\title{
D3-Branes Wrapped on a Spindle
}

\author{
Pietro Ferrero $\odot,{ }^{1}$ Jerome P. Gauntlett๑, ${ }^{2}$ Juan Manuel Pérez Ipiña $\odot,{ }^{1}$ Dario Martelli® ${ }^{3,4}$ and James Sparks ${ }^{1}$ \\ ${ }^{1}$ Mathematical Institute, University of Oxford, Woodstock Road, Oxford OX2 6GG, United Kingdom \\ ${ }^{2}$ Blackett Laboratory, Imperial College, Prince Consort Road, London SW7 2AZ, United Kingdom \\ ${ }^{3}$ Dipartimento di Matematica, Università di Torino, Via Carlo Alberto 10, 10123 Torino, Italy \\ ${ }^{4}$ INFN, Sezione di Torino \& Arnold-Regge Center, Via Pietro Giuria 1, 10125 Torino, Italy
}

(Received 22 December 2020; accepted 19 February 2021; published 18 March 2021)

\begin{abstract}
We construct supersymmetric $\mathrm{AdS}_{3} \times \Sigma$ solutions of minimal gauged supergravity in $D=5$, where $\Sigma$ is a two-dimensional orbifold known as a spindle. Remarkably, these uplift on $S^{5}$, or more generally on any regular Sasaki-Einstein manifold, to smooth solutions of type IIB supergravity. The solutions are dual to $d=2, \mathcal{N}=(0,2)$ SCFTs and we show that the central charge for the gravity solution agrees with a field theory calculation associated with D3-branes wrapped on $\Sigma$.
\end{abstract}

DOI: 10.1103/PhysRevLett.126.111601

Introduction.-Important insights into strongly coupled supersymmetric conformal field theories (SCFTs) can be obtained by realizing them as the renormalization group fixed points of compactifications of higher-dimensional field theories. Such SCFTs can be constructed by starting with the field theories arising on the world volumes of branes in string theory or $\mathrm{M}$ theory, wrapping them on a compact manifold $\Sigma$, and then flowing to the infrared (IR).

In favorable circumstances these configurations can be described within the AdS/CFT correspondence by brane solutions of $D=10 / 11$ supergravity. Such solutions have a boundary of the form $\operatorname{AdS}_{d+1} \times M$, where $M$ is a compact manifold, which describes the ultraviolet (UV) of the SCFT in $d$ spacetime dimensions. They also have near horizon geometries of the schematic form $\operatorname{AdS}_{d+1-n} \times \Sigma \times M$, which describes the SCFT in $d-n$ dimensions arising in the IR, where $\Sigma$ has dimension $n$. After a Kaluza-Klein reduction of the $D=10 / 11$ supergravity theory on $M$, to obtain a gravity theory in $d+1$ spacetime dimensions, these solutions may be viewed as black branes in $\operatorname{AdS}_{d+1}$ with horizon $\Sigma$.

Such solutions were first constructed in the foundational work [1] and describe M5-branes and D3-branes wrapping Riemann surfaces of constant curvature, with genus $g>1$. Subsequently there have been many generalizations including wrapping manifolds of higher dimension, relaxing the constant curvature condition, allowing for punctures, etc. (e.g., Refs. [2-4]). In all these developments, supersymmetry is preserved by demanding that the field theory

Published by the American Physical Society under the terms of the Creative Commons Attribution 4.0 International license. Further distribution of this work must maintain attribution to the author(s) and the published article's title, journal citation, and DOI. Funded by SCOAP. arising on the brane world volume is "topologically twisted" $[5,6]$. This involves a specific coupling both to the metric on $\Sigma$ and to external R-symmetry gauge fields. An important consequence of this twisting is that the Killing spinors preserved by the solution are independent of the coordinates of $\Sigma$.

Here we discuss a class of supersymmetric solutions which have fundamentally new features. We present $\mathrm{AdS}_{3} \times \Sigma$ solutions of $D=5$ minimal gauged supergravity which we interpret as the near horizon limit of black brane solutions associated with D3-branes wrapped on $\Sigma$. The first new feature is that supersymmetry is not realized by a topological twist. The second is that $\Sigma$ is not a compact manifold but an orbifold [7]. More specifically, we will consider the weighted projective space $\Sigma=\mathbb{W C P}_{\left[n_{-}, n_{+}\right]}^{1}$, also known as a spindle. This is topologically a twosphere but with conical deficit angles $2 \pi\left(1-1 / n_{\mp}\right)$ at the poles, specified by two coprime positive integers $n_{\mp}$, with $n_{-} \neq n_{+}[8]$.

Remarkably, after uplifting the $\mathrm{AdS}_{3} \times \Sigma$ solutions on specific Sasaki-Einstein five-manifolds, $\mathrm{SE}_{5}$, to obtain solutions of type IIB supergravity, they become completely smooth [9]. Moreover, the resulting $\mathrm{AdS}_{3} \times \mathcal{M}_{7}$ solutions are precisely those of Ref. [11]. Our construction suggests that the $d=2, \mathcal{N}=(0,2)$ SCFTs dual to the $\mathrm{AdS}_{3} \times \mathcal{M}_{7}$ solutions of Ref. [11] arise from compactifying $d=4$, $\mathcal{N}=1$ SCFTs on a spindle, where the $d=4$ theories are dual to $\mathrm{AdS}_{5} \times \mathrm{SE}_{5}$. This includes the case of $\mathcal{N}=4$ $\mathrm{SYM}$, where $\mathrm{SE}_{5}=S^{5}$. We make a precision test of this interpretation: we compute the central charge and superconformal R-symmetry of the $d=2$ field theories using anomaly polynomials and $c$ extremization [12] and find exact agreement with the gravity result [11].

$D=5$ solutions. - The equations of motion for $D=5$ minimal gauged supergravity [13] are given by 


$$
\begin{aligned}
R_{\mu \nu} & =-4 g_{\mu \nu}+\frac{2}{3} F_{\mu \rho} F_{\nu}^{\rho}-\frac{1}{9} g_{\mu \nu} F_{\rho \sigma} F^{\rho \sigma}, \\
d * F & =-\frac{2}{3} F \wedge F,
\end{aligned}
$$

where $F=d A$, with $A$ the Abelian R-symmetry gauge field. A solution is supersymmetric if it admits a Killing spinor satisfying

$$
\left[\nabla_{\mu}-\frac{\mathrm{i}}{12}\left(\Gamma_{\mu}^{\nu \rho}-4 \delta_{\mu}^{\nu} \Gamma^{\rho}\right) F_{\nu \rho}-\frac{1}{2} \Gamma_{\mu}-\mathrm{i} A_{\mu}\right] \epsilon=0,
$$

where $\epsilon$ is a Dirac spinor and $\left\{\Gamma_{\mu}, \Gamma_{\nu}\right\}=2 g_{\mu \nu}$.

The supersymmetric solution of interest is given by

$$
d s_{5}^{2}=\frac{4 y}{9} d s_{\mathrm{AdS}_{3}}^{2}+d s_{\Sigma}^{2}, \quad A=\frac{1}{4}\left(1-\frac{a}{y}\right) d z .
$$

Here $d s_{\mathrm{AdS}_{3}}^{2}$ is a unit radius metric on $\mathrm{AdS}_{3}$, while

$$
d s_{\Sigma}^{2}=\frac{y}{q(y)} d y^{2}+\frac{q(y)}{36 y^{2}} d z^{2},
$$

is the metric on the horizon $\Sigma$ and

$$
q(y)=4 y^{3}-9 y^{2}+6 a y-a^{2},
$$

with $a$ a constant.

Assuming $a \in(0,1)$ the three roots $y_{i}$ of $q(y)$ are all real and positive. Defining $y_{1}<y_{2}<y_{3}$, we then take $y \in$ $\left[y_{1}, y_{2}\right]$ to obtain a positive definite metric (4) on $\Sigma$. However, as $y$ approaches $y_{1}$ and $y_{2}$ it is not possible to remove the conical deficit singularities at both roots by a single choice of period $\Delta z$ for $z$, to obtain a smooth twosphere. Instead we find that if

$$
\begin{aligned}
a & =\frac{\left(n_{-}-n_{+}\right)^{2}\left(2 n_{-}+n_{+}\right)^{2}\left(n_{-}+2 n_{+}\right)^{2}}{4\left(n_{-}^{2}+n_{-} n_{+}+n_{+}^{2}\right)^{3}}, \\
\Delta z & =\frac{2\left(n_{-}^{2}+n_{-} n_{+}+n_{+}^{2}\right)}{3 n_{-} n_{+}\left(n_{-}+n_{+}\right)} 2 \pi,
\end{aligned}
$$

then $d s_{\Sigma}^{2}$ is a smooth metric on the orbifold $\Sigma=\mathbb{W} \mathbb{C P} \mathbb{P}_{\left[n_{-}, n_{+}\right]}^{1}$. Specifically, there are conical deficit angles $2 \pi\left(1-1 / n_{\mp}\right)$ at $y=y_{1}, y_{2}$, respectively, where $n_{ \pm}$are arbitrary coprime positive integers with $n_{-}>n_{+}$.

Note that there is magnetic flux through $\Sigma$ :

$$
\frac{1}{2 \pi} \int_{\Sigma} F=\frac{n_{-}-n_{+}}{2 n_{-} n_{+}} .
$$

This may be contrasted with the Euler number

$$
\chi(\Sigma)=\frac{1}{4 \pi} \int_{\Sigma} R_{\Sigma} \operatorname{vol}_{\Sigma}=\frac{n_{-}+n_{+}}{n_{-} n_{+}},
$$

where $R_{\Sigma}$ is the Ricci scalar of $\Sigma$, and $\mathrm{vol}_{\Sigma}$ is its volume form [14].

To solve Eq. (2) we write $\Gamma^{a}=\gamma^{a} \otimes \sigma^{3}$, for $a=0,1,2$ with $\gamma^{0}=-\mathrm{i} \sigma^{2}, \quad \gamma^{1}=\sigma^{1}, \quad \gamma^{2}=\sigma^{3}$, and $\Gamma^{3}=1 \otimes \sigma^{2}$, $\Gamma^{4}=1 \otimes \sigma^{1}$, where $\sigma^{i}$ are Pauli matrices. We then write $\epsilon=\vartheta \otimes \chi$ with $\vartheta$ a Killing spinor for $\mathrm{AdS}_{3}$ satisfying $\nabla_{a} \vartheta=\frac{1}{2} \gamma_{a} \vartheta$. The two-component spinor $\chi$ on the spindle is given by

$$
\chi=\left(\frac{\sqrt{q_{1}(y)}}{\sqrt{y}}, \mathrm{i} \frac{\sqrt{q_{2}(y)}}{\sqrt{y}}\right)
$$

where

$$
q_{1}(y)=-a+2 y^{3 / 2}+3 y, \quad q_{2}(y)=a+2 y^{3 / 2}-3 y,
$$

which satisfy $q(y)=q_{1}(y) q_{2}(y)$. In contrast to the topological twist, this spinor depends on the coordinates of $\Sigma$. Moreover, as shown in Ref. [15], the spinor is in fact a section of a nontrivial bundle over $\Sigma$. Note that the gauge choice used in Eq. (3) has been fixed by requiring $\epsilon$ to be independent of $z$.

Uplift to IIB string theory.-Any supersymmetric solution to Eq. (1) uplifts (locally) to type IIB supergravity via [16]:

$$
\begin{aligned}
d s_{10}^{2}= & L^{2}\left[d s_{5}^{2}+\left(\frac{1}{3} d \psi+\sigma+\frac{2}{3} A\right)^{2}+d s_{K E_{4}}^{2}\right] \\
g_{s} F_{5}= & L^{4}\left[4 \mathrm{vol}_{5}-\frac{2}{3} *_{5} F \wedge J\right. \\
& \left.+\left(2 J \wedge J-\frac{2}{3} F \wedge J\right) \wedge\left(\frac{1}{3} d \psi+\sigma+\frac{2}{3} A\right)\right] .
\end{aligned}
$$

Here $F_{5}$ is the self-dual five-form, $g_{s}$ is the string coupling constant, and $L>0$ is a length scale that is fixed by flux quantization. $\mathrm{KE}_{4}$ is an arbitrary positively curved KählerEinstein four-manifold with Kähler form $J$, normalized so that the Ricci form is $\mathcal{R}=6 J$, and $\sigma$ is a local one-form with $d \sigma=2 J$.

Substituting Eq. (3) into Eq. (11) we find that the $D=10$ metric may be written as

$$
d s_{10}^{2}=\frac{4}{9} L^{2} y\left[d s_{\mathrm{AdS}_{3}}^{2}+d s_{\mathcal{M}_{7}}^{2}\right],
$$

where $\mathcal{M}_{7}$ is a compact seven-manifold. This is the same solution of type IIB supergravity given in Ref. [11].

It was shown in Ref. [11] that $\mathcal{M}_{7}$ is the total space of a lens space $S^{3} / \mathbb{Z}_{q}$ fibration over the $\mathrm{KE}_{4}$, where the twisting is parametrized by another positive integer $p$. The lens space fiber has coordinates $y, z, \psi$. In terms of our parameters $n_{ \pm}$we identify 


$$
p=k n_{+}, \quad q=\frac{k}{I}\left(n_{-}-n_{+}\right),
$$

where $p, q \in \mathbb{N}$ are coprime. The Fano index $I$ is the largest positive integer for which $\int_{S} c_{1} / I \in \mathbb{Z}$, for all two-cycles $S$ in the $\mathrm{KE}_{4}$, where $c_{1}=[\mathcal{R} / 2 \pi] \in H^{2}\left(\mathrm{KE}_{4}, \mathbb{Z}\right)$. We have also defined

$$
k=\operatorname{hcf}(I, p),
$$

and identify $\psi$ with period $\Delta \psi$ given by [17]

$$
\Delta \psi=\frac{2 \pi I}{k} .
$$

At a fixed point in the $D=5$ spacetime, the internal five-dimensional metric $d s_{\mathrm{SE}_{5}}^{2}=\left(\frac{1}{3} d \psi+\sigma\right)^{2}+d s_{\mathrm{KE}_{4}}^{2}$ in Eq. (11) is then a regular Sasaki-Einstein manifold, which is simply connected when $k=1$.

To obtain a string theory background one must also quantize the five-form flux $F_{5}$ through all five-cycles in $\mathcal{M}_{7}$. This was carried out in Ref. [11]. We define the integers

$$
M=\int_{\mathrm{KE}_{4}} c_{1} \wedge c_{1}=\frac{1}{4 \pi^{2}} \int_{\mathrm{KE}_{4}} \mathcal{R} \wedge \mathcal{R},
$$

and $h=\operatorname{hcf}\left(M / I^{2}, q\right)$. Then if we choose $L$ to satisfy

$$
\frac{L^{4}}{g_{s} \ell_{s}^{4}}=\frac{108 \pi}{I^{3} h} k^{2} n_{+} n_{-} n,
$$

where $\ell_{s}$ is the string length and $n \in \mathbb{N}$, then one finds that $1 /\left(2 \pi \ell_{s}\right)^{4} \int_{D} F_{5} \in \mathbb{Z}$, for all five-cycles $D$ in $\mathcal{M}_{7}$.

There is a finite set of choices for the positively curved $\mathrm{KE}_{4}$. If $\mathrm{KE}_{4}=\mathbb{C P}^{2}$ then $I=3, M=9$. For this case, $k=1$ gives $\mathrm{SE}_{5}=S^{5}$ as the internal space while $k=3$ gives $S^{5} / \mathbb{Z}_{3}$. If $\mathrm{KE}_{4}=S^{2} \times S^{2}$ we have $I=2, M=8$. Now $k=1$ gives $\mathrm{SE}_{5}=T^{1,1}$, while $k=2$ gives $\mathrm{SE}_{5}=T^{1,1} / \mathbb{Z}_{2}$. Finally, for $\mathrm{KE}_{4}=d P_{m}, \quad 3 \leq m \leq 8$, where $d P_{m}$ is a del Pezzo surface, we have $I=1$, $M=9-m$.

A key observation is that $\mathcal{M}_{7}$ in the $\mathrm{AdS}_{3}$ solutions of Ref. [11] may also be viewed as $\mathrm{SE}_{5}$ fibrations over $\Sigma=\mathbb{W C P}_{\left[n_{-}, n_{+}\right]}^{1}$. We can begin with any weighted projective space, with weights $n_{-}>n_{+}$, and then define $p, q \in \mathbb{Z}$ via (13), where we also define

$$
k=\frac{I}{\operatorname{hcf}\left(I, n_{-}-n_{+}\right)} .
$$

With this definition, $p$ and $q$ are manifestly coprime, and one can check that Eq. (14) is equivalent to Eq. (18). With this perspective, we can calculate the flux of $F_{5}$ through the $\mathrm{SE}_{5}$ fiber:

$$
N \equiv \frac{1}{\left(2 \pi \ell_{s}\right)^{4}} \int_{\mathrm{SE}_{5}} F_{5}=\frac{M}{I^{2} h} k n_{+} n_{-} n \in \mathbb{N} .
$$

Notice that for a given spindle, specified by $n_{ \pm}$, and a given choice of $\mathrm{KE}_{4}$ we only get a smooth type IIB solution for $k$ as in Eq. (18) and hence a specific $\mathrm{SE}_{5}$. E.g., if $\mathrm{KE}_{4}=\mathbb{C P}^{2}$ and $n_{+}=2$, then for $n_{-}=3,7, \ldots$ and $n_{-}=5,9, \ldots$ we can uplift on $S^{5} / \mathbb{Z}_{3}$ and $S^{5}$, respectively.

The central charge is given by $c=3 L / 2 G_{(3)}$, where $G_{(3)}$ is the Newton constant obtained by compactifying type IIB supergravity on $\mathcal{M}_{7}$ [18]. We can rewrite the result of Ref. [11] as

$$
c=\frac{4\left(n_{-}-n_{+}\right)^{3}}{3 n_{-} n_{+}\left(n_{-}^{2}+n_{-} n_{+}+n_{+}^{2}\right)} a_{4 d},
$$

where

$$
a_{4 d} \equiv \frac{\pi^{2} N^{2}}{4 \operatorname{vol}\left(\mathrm{SE}_{5}\right)}
$$

In Ref. [11] the dual $d=2, \mathcal{N}=(0,2)$ SCFTs were not identified, but our $D=5$ construction of the solutions, together with the flux condition (19), leads to a conjecture. Begin with the $d=4 \mathrm{SCFT}$ dual to $\mathrm{AdS}_{5} \times \mathrm{SE}_{5}$, describing $N$ D3-branes at the Calabi-Yau threefold singularity with conical metric $d r^{2}+r^{2} d s_{\mathrm{SE}_{5}}^{2}$. The large $N a$-central charge of this theory is precisely given by $a_{4 d}$ in Eq. (21) [19]. One then compactifies that theory on $\Sigma=\mathbb{W C P}_{\left[n_{-}, n_{+}\right]}^{1}$, with a background R-symmetry gauge field with magnetic flux (7). The solutions we have described suggest the theory flows to a $d=2, \mathcal{N}=(0,2)$ SCFT in the IR, and we will give evidence for this below by computing the central charge via a field theory calculation.

The $U(1)_{R}$ symmetry of the $(0,2)$ theory dual to the $\mathrm{AdS}_{3} \times \mathcal{M}_{7}$ solutions is realized by a Killing vector, $R_{2 d}$, on $\mathcal{M}_{7}$. Using the results of Refs. $[20,21]$ we deduce

$$
R_{2 d}=2 \partial_{\psi}+\frac{3 n_{-} n_{+}\left(n_{-}+n_{+}\right)}{n_{-}^{2}+n_{-} n_{+}+n_{+}^{2}} \partial_{\varphi} .
$$

Here we have defined $\varphi=\frac{2 \pi z}{\Delta z}$ so that $\Delta \varphi=2 \pi$, and $\partial_{\varphi}$ generates the $U(1)$ isometry of the weighted projective space $\Sigma$, which we shall refer to as $U(1)_{J}$. Note that the Killing spinor on the $\mathrm{SE}_{5}$ has unit charge under $R_{4 d}=2 \partial_{\psi}$, which may therefore be identified with the superconformal $U(1)_{R}$ symmetry of the $d=4$ SCFT before compactification on $\Sigma$. In other words, Eq. (22) states that the $d=4$ R-symmetry mixes with $U(1)_{J}$ in flowing to the $d=2$ R-symmetry in the IR. We shall also recover Eq. (22) from a field theory calculation in the next section.

$d=4$ SCFTs on $\Sigma$.-We begin with a general $d=4$ SCFT with anomaly polynomial given by the 6 -form 


$$
\mathcal{A}_{4 d}=\frac{\operatorname{tr} R^{3}}{6} c_{1}\left(R_{4 d}\right)^{3}-\frac{\operatorname{tr} R}{24} c_{1}\left(R_{4 d}\right) p_{1}\left(T Z_{6}\right) .
$$

As is standard, in (even) dimension $d$ the anomaly polynomial is a $(d+2)$-form on an abstract $(d+2)$ dimensional space, here called $Z_{6} \cdot c_{1}\left(R_{4 d}\right)$ denotes the first Chern class of the $d=4$ superconformal $U(1)_{R}$ symmetry bundle over $Z_{6}$, and $p_{1}$ denotes the first Pontryagin class. The trace is over Weyl fermions when the theory has a Lagrangian description, and in any case we may always write

$\operatorname{tr} R^{3}=\frac{16}{9}\left(5 a_{4 d}-3 c_{4 d}\right), \quad \operatorname{tr} R=16\left(a_{4 d}-c_{4 d}\right)$,

in terms of the central charges $a_{4 d}, c_{4 d}$. We focus on the large $N$ limit in which $a_{4 d}=c_{4 d}$ to leading order, and hence

$$
\mathcal{A}_{4 d}=\frac{16 a_{4 d}}{27} c_{1}\left(R_{4 d}\right)^{3} \quad(\text { at large } N) .
$$

We now compactify the $d=4$ theory on $\Sigma=\mathbb{W C P}_{\left[n_{-}, n_{+}\right]}^{1}$, with magnetic flux (7) for the $d=4$ R-symmetry gauge field. The resulting $d=2$ anomaly polynomial will then capture the right-moving central charge $c_{r}$, but crucially we need to include the $U(1)_{J}$ global symmetry in $d=2$ that comes from the isometry of $\Sigma$. Geometrically, this involves taking $Z_{6}$ to be the total space of a $\Sigma$ fibration over a fourmanifold $Z_{4}$ [22]. More precisely, we let $J$ be a $U(1)$ bundle over $Z_{4}$, with connection corresponding to a background gauge field $A_{J}$ for the $d=2 U(1)_{J}$ global symmetry, and then fiber $\Sigma$ over $Z_{4}$ using the $U(1)_{J}$ action and connection $A_{J}$. In practice, this amounts to the replacement $d \varphi \mapsto d \varphi+A_{J}$.

Incorporating the magnetic flux (7) into this construction amounts to "gauging" the $U(1)$ gauge field $A$ in Eq. (3), as just described. Thus, we define the following connection one-form on $Z_{6}$ :

$$
\mathcal{A}=\frac{1}{4}\left(1-\frac{a}{y}\right) \frac{\Delta z}{2 \pi}\left(d \varphi+A_{J}\right) \equiv \rho(y)\left(d \varphi+A_{J}\right),
$$

where recall that the gauge choice we made is such that the Killing spinors are uncharged under the $U(1)_{J}$ symmetry generated by $\partial_{\varphi}$. This is necessary for the twisting to make sense. $\mathcal{A}$ defined by Eq. (26) is a gauge field on $Z_{6}$, which restricts to the supergravity gauge field $A$ in Eq. (3) on each $\Sigma$ fiber. We compute the curvature

$$
\mathcal{F}=d \mathcal{A}=\rho^{\prime}(y) d y \wedge\left(d \varphi+A_{J}\right)+\rho(y) F_{J},
$$

where $F_{J}=d A_{J}$. The one-form $d \varphi+A_{J}$ is precisely the global angular form for the $U(1)$ bundle, and so is globally defined on $Z_{6}$ away from the poles of $\Sigma=\mathbb{W C P}_{\left[n_{-}, n_{+}\right]}^{1}$ at $y=y_{1}, y_{2}$. Moreover, one can verify that $\rho^{\prime}(y) d y \wedge d \varphi$ vanishes smoothly at the poles, where the angular coordinate $\varphi$ is not defined, implying that $\mathcal{F}$ is a globally defined closed two-form on $Z_{6}$. By construction, the integral of $\mathcal{F}$ over a fiber $\Sigma$ of $Z_{6}$ satisfies Eq. (7). More generally, the integrals of wedge products over the fibers are given, for $s \in \mathbb{N}$, by

$$
\int_{\Sigma}\left(\frac{\mathcal{F}}{2 \pi}\right)^{s}=\frac{1}{2^{s}}\left(\frac{1}{n_{+}^{s}}-\frac{1}{n_{-}^{s}}\right)\left(-\frac{F_{J}}{2 \pi}\right)^{s-1} .
$$

The curvature form $\mathcal{F}$ defines a $U(1)$ bundle $\mathcal{L}$ over $Z_{6}$ by taking $c_{1}(\mathcal{L})=[\mathcal{F} / 2 \pi] \in H^{2}\left(Z_{6}, \mathbb{R}\right)$. This is different from Ref. [22], where the $U(1)$ bundle was taken to be the tangent bundle to the fibers $T_{\text {fibers }} Z_{6}$, which gives the Euler class (8), rather than Eq. (7). We note that at the poles we have $\left.c_{1}(\mathcal{L})\right|_{y=y_{1}, y_{2}}=-\frac{1}{2 n_{ \pm}} c_{1}(J)$, where we have defined $c_{1}(J)=\left[F_{J} / 2 \pi\right] \in H^{2}\left(Z_{4}^{ \pm}, \mathbb{Z}\right)$. In the anomaly polynomial we then write

$$
c_{1}\left(R_{4 d}\right)=c_{1}\left(R_{2 d}\right)+c_{1}(\mathcal{L}),
$$

where $R_{2 d}$ is the pullback of a $U(1)$ bundle over $Z_{4}$. Notice that the twisting (29) will make sense globally only if the $d=4 R$ charges of fields satisfy appropriate quantization conditions, and for gauge-invariant operators this is equivalent to the global regularity and flux quantization conditions imposed on the supergravity solutions, cf. the discussion below Eq. (19).

The $d=2$ anomaly polynomial is obtained by integrating $\mathcal{A}_{4 d}$ in Eq. (25) over $\Sigma$. Using Eqs. (28) and (29) we compute

$$
\begin{aligned}
\mathcal{A}_{2 d}= & \frac{2 a_{4 d}}{27}\left[12\left(\frac{1}{n_{+}}-\frac{1}{n_{-}}\right) c_{1}\left(R_{2 d}\right)^{2}\right. \\
& \left.-6\left(\frac{1}{n_{+}^{2}}-\frac{1}{n_{-}^{2}}\right) c_{1}\left(R_{2 d}\right) c_{1}(J)+\left(\frac{1}{n_{+}^{3}}-\frac{1}{n_{-}^{3}}\right) c_{1}(J)^{2}\right] .
\end{aligned}
$$

The coefficient of $\frac{1}{2} c_{1}\left(L_{i}\right) c_{1}\left(L_{j}\right)$ in $\mathcal{A}_{2 d}$ is $\operatorname{tr} \gamma^{3} Q_{i} Q_{j}$, where the global symmetry $Q_{i}$ is associated to the $U(1)$ bundle $L_{i}$ over $Z_{4}$, and $\gamma^{3}$ is the $d=2$ chirality operator. On the other hand, $c$ extremization [12] implies that the $d=2$ superconformal $U(1)_{R}$ extremizes

$$
c_{\text {trial }}=3 \operatorname{tr} \gamma^{3} R_{\text {trial }}^{2},
$$

over the space of possible R-symmetries. We set

$$
R_{\text {trial }}=R_{2 d}+\varepsilon J,
$$

and extremize the quadratic function of $\varepsilon$ one obtains from Eqs. (30) and (31). The extremal value is 


$$
\varepsilon_{*}=\frac{3 n_{-} n_{+}\left(n_{-}+n_{+}\right)}{n_{-}^{2}+n_{-} n_{+}+n_{+}^{2}} .
$$

The right-moving central charge is given by Eq. (31) evaluated on the superconformal R-symmetry [12]. Substituting Eq. (33) into Eqs. (31), (32) we find

$$
c_{r}=\frac{\left(n_{-}-n_{+}\right)^{3}}{n_{-} n_{+}\left(n_{-}^{2}+n_{-} n_{+}+n_{+}^{2}\right)} \frac{4 a_{4 d}}{3} .
$$

At leading order in $N, c_{r}=c_{l} \equiv c$ is the central charge of the SCFT, and we see that the field theory result (34) precisely matches the gravity result (20). Moreover, the R-symmetry (32), with $\varepsilon=\varepsilon_{*}$, precisely matches the supergravity R-symmetry (22).

Discussion.-Our solutions exhibit a number of new properties, raising several directions for future research. First, despite having orbifold singularities in $D=5$, when uplifted to $D=10$ the solutions are completely regular. What type of singularities are permitted in lowerdimensional supergravity theories that have this property? Second, it is often claimed that supersymmetry requires a topological twist when branes wrap a compact manifold, so that the "twisted spinors" are constant on the manifold. Our near horizon solutions are a counterexample and it would be interesting to understand this more systematically. Third, our results suggest there should exist black string solutions which approach $\mathrm{AdS}_{5}$ in the UV and $\mathrm{AdS}_{3} \times \Sigma$ in the IR. Such solutions will reveal the precise deformations of the $d=4, \mathcal{N}=1$ SCFTs that can then flow to the $d=2, \mathcal{N}=(0,2)$ SCFTs dual to the $\mathrm{AdS}_{3} \times \mathcal{M}_{7}$ solutions of Ref. [11], including the way in which the D3-brane wrapping the spindle is preserving supersymmetry.

In Ref. [15] we present analogous supergravity solutions in $D=4$, associated with M2-branes wrapped on a "spinning spindle." In that case the full black hole solution is known and it approaches $\mathrm{AdS}_{4}$ in the UV and $\mathrm{AdS}_{2} \times \Sigma$ in the IR, with a spindle horizon $\Sigma$. The $D=4$ black hole is accelerating and this leads to the conical deficit singularities on $\Sigma$. Once lifted to $D=11$ these orbifold singularities are removed and the solutions become completely regular. Supersymmetry is again not realized by the topological twist for the $\mathrm{AdS}_{2} \times \Sigma$ solution, similar to our $\mathrm{AdS}_{3} \times \Sigma$ solutions. In the UV, the black holes at finite temperature have a conformal boundary consisting of a spindle. In the supersymmetric and extremal limit, however, the spindle degenerates into "two halves," each of which is associated with a topological twist, but with a different constant spinor on each component. It is another fascinating open question to determine how typical such a novel realization of supersymmetry is for branes wrapping spindles, as well as spaces of higher dimension.

Our results also suggest many questions on the field theory side. When defining a SCFT on a spindle what additional data should be specified at the orbifold points?
Considering weakly coupled $\mathcal{N}=4$ SYM theory would be an important first step. Our holographic analysis shows that SCFTs dual to regular SE manifolds can only be placed on certain spindles and there is an apparent obstruction for those dual to irregular SE manifolds; why is this? The anomaly polynomial technique we employed is based on smooth manifolds and yet it gives a consistent result in the context of the orbifolds we studied, in the large $N$ limit. It would be interesting to justify this more systematically (also see Ref. [23]) and determine subleading contributions.

We thank K. Hristov and J. Lucietti for comments. This work was supported in part by STFC Grants No. ST/ P000762/1, No. ST/T000791/1 and No. ST/T000864/1. J.P. G. is supported as a KIAS Scholar and as a Visiting Fellow at the Perimeter Institute.

[1] J. M. Maldacena and C. Nunez, Int. J. Mod. Phys. A 16, 822 (2001).

[2] J. P. Gauntlett, N. Kim, and D. Waldram, Phys. Rev. D 63, 126001 (2001).

[3] D. Gaiotto and J. Maldacena, J. High Energy Phys. 10 (2012) 189.

[4] M. T. Anderson, C. Beem, N. Bobev, and L. Rastelli, Commun. Math. Phys. 318, 429 (2013).

[5] E. Witten, Commun. Math. Phys. 117, 353 (1988).

[6] M. Bershadsky, C. Vafa, and V. Sadov, Nucl. Phys. B463, 420 (1996).

[7] An orbifold is locally modelled on open subsets of $\mathbb{R}^{n} / \Gamma$, where $\Gamma$ are finite groups.

[8] $\mathbb{W C P}_{\left[n_{-}, n_{+}\right]}^{1}$ is a bad orbifold in the sense that it is not possible to move to a covering space that is a manifold. It does not admit a metric of constant curvature.

[9] The local $D=5$ solutions were independently presented in Ref. [10], who also made a local connection with the solutions of Ref. [11].

[10] H. K. Kunduri, J. Lucietti, and H. S. Reall, J. High Energy Phys. 02 (2007) 026.

[11] J. P. Gauntlett, O. A. P. Mac Conamhna, T. Mateos, and D. Waldram, Phys. Rev. Lett. 97, 171601 (2006).

[12] F. Benini and N. Bobev, Phys. Rev. Lett. 110, 061601 (2013).

[13] M. Gunaydin, G. Sierra, and P. Townsend, Nucl. Phys. B242, 244 (1984).

[14] In general the integral of the curvature for a $U(1)$ gauge field on $\mathbb{W C P}_{\left[n_{-}, n_{+}\right]}^{1}$ is necessarily $2 \pi /\left(n_{-} n_{+}\right)$times an integer e.g., [15]. This makes the gauge field $A$, with flux given by (7), a spin ${ }^{c}$ gauge field: $n_{-}-n_{+}$is even/odd precisely when $n_{-}+n_{+}$is even/odd, so that spinor fields with unit charge under $A$ are always globally well-defined.

[15] P. Ferrero, J. P. Gauntlett, J. M. P. Ipiña, D. Martelli, and J. Sparks, arXiv:2012.08530.

[16] A. Buchel and J. T. Liu, Nucl. Phys. B771, 93 (2007).

[17] The torus parametrised by $(z, \psi)$ is the same as [11]. We have made identifications on $z$ and $\psi$ in the opposite order to [11], so (15) is not the same but $\Delta \psi \Delta z$ is.

[18] J. Brown and M. Henneaux, Commun. Math. Phys. 104, 207 (1986). 
[19] S. S. Gubser, Phys. Rev. D 59, 025006 (1999).

[20] N. Kim, J. High Energy Phys. 01 (2006) 094.

[21] J. P. Gauntlett, N. Kim, and D. Waldram, J. High Energy Phys. 04 (2007) 005.
[22] S. M. Hosseini, K. Hristov, Y. Tachikawa, and A. Zaffaroni, J. High Energy Phys. 09 (2020) 167.

[23] I. Bah, F. Bonetti, R. Minasian, and P. Weck, J. High Energy Phys. 02 (2021) 116. 